\title{
Bunch length modulation in highly dispersive storage rings
}

\author{
C. Biscari \\ Laboratori Nazionali di Frascati, INFN, Frascati, C.P. 13, 00044, Italy
}

(Received 7 June 2005; published 12 September 2005)

\begin{abstract}
Modulation of the bunch length in an electron storage ring appears with the combination of a high rf voltage derivative and large dispersion in the dipoles, producing drifting of the longitudinal phase plane along the closed orbit. The comparison is done between two different regimes: the one corresponding to high momentum compaction structures, in which the drifting in all dipoles has always the same direction, and the other one corresponding to low momentum compaction in which the drifting changes sign along the ring. Expressions for the longitudinal Twiss functions, energy spread and longitudinal emittance are given. The generalization to multiple $\mathrm{rf}$ systems is mentioned.
\end{abstract}

DOI: 10.1103/PhysRevSTAB.8.091001

PACS numbers: 29.20.Dh, 29.27.Bd

\section{INTRODUCTION}

Obtaining very short bunches in $\mathrm{e}^{+}$and $\mathrm{e}^{-}$storage rings is an issue for colliders and synchrotron light sources in the path for high peak currents. The natural bunch length is determined by the rf system parameters and by the momentum compaction $\alpha_{c}$, while the anomalous bunch lengthening above the microwave instability threshold can dominate the bunch longitudinal dimensions, specially at low energies. In the last generation of rings the bunch length has reached values of few millimeters, thanks to the advanced design of very low impedance vacuum chambers and to the high voltage rf systems. Going to the $\mathrm{mm}$ range with high currents asks for further developments.

The bunch length modulation along the ring can be one of them. Already in the far 1969 Piwinski had derived the expressions for the longitudinal motion with high synchrotron tune which correspond to bunch length variation along the ring [1]. During the last LEP runs Hofmann had made a proposal to make an experiment on bunch length modulation [2], but the priority was LEP dismantling and the experiment remained in Albert's mind. Recently, in the framework of superfactories studies, the strong rf focusing regime has been proposed [3] to modulate the bunch length along the ring in order to obtain short bunches in a limited zone of a collider. This regime requires both a high rf voltage and a high momentum compaction lattice. The resulting high synchrotron tune has the drawback of the critical choice of the working point in three dimensions, which may affect dynamic aperture, beam-beam behavior and beam lifetime [4].

An evolution of this principle is described in the following, where the ring lattice has a strong correlation between particle longitudinal position and energy deviation, but with opposite signs on different zones of the ring, resulting in a small momentum compaction and therefore low synchrotron tune.

The generalization to multiple rf systems is also mentioned.

\section{LONGITUDINAL OSCILLATION IN A RING WITH ONE RF CAVITY}

The single particle longitudinal dynamics in electron storage rings is usually described in the small amplitude oscillation approximation [5]. A more general description is needed for regimes implying large excursions in the longitudinal phase space.

Let us recall some well known expressions which will be useful for the following considerations. The coordinates describing the particle motion along the ring are the 6 elements of the vector:

$$
\left(x, p_{x}, y, p_{y}, c t, \delta E / E\right)
$$

where the first four elements describe the transverse planes, $c t$ is the longitudinal position with respect to the synchronous particle, and $\delta E / E$ the energy deviation. The action of the ring elements on the particle motion is described by $6 \times 6$ transport matrices. The $6 \times 6 \mathbf{R}$ one-turn matrix in a point $s$ of the ring is the product of all the elements $6 \times 6$ transfer matrix from $s$ to $s+L$, where $L$ is the ring length:

$$
\mathbf{R}(s)=\prod_{s}^{s+L} \mathbf{R}^{i}
$$

with $\mathbf{R}^{i}$ the transfer matrix of the $i$ th element. The $\mathrm{rf}$ cavities are also included in the above expression, even if usually their effect is very weak and negligible.

The elements of $\mathbf{R}(s)$ are functions of the azimuth $s$ along the ring. From their value the Twiss functions and the total phase advance are defined. While the Twiss functions are periodic with $L$, the phase advance is a parameter of the ring. This is very often used for the transverse planes, while less used for the longitudinal one, since the variation along the ring of the longitudinal Twiss functions is usually very small and therefore neglected.

Only in dipoles the term $R_{56}^{i}$, relating the particle longitudinal position along the bunch to its energy deviation, is different from zero, and corresponds to the integral of the 
dispersion function, $D(s)$, divided by the bending radius $\rho(s)$ along the dipole in the hypothesis of $D(s)=D^{\prime}(s)=$ 0 at one end of the dipole.

In a transfer line, whose initial point is dispersion free, the term $R^{T}{ }_{56}$ of the total transport matrix can be obtained both by multiplying all the element transport matrices (including also elements where the $R_{56}$ term is zero) or by applying:

$$
R^{T}{ }_{56}=-\sum_{i} \int_{0}^{l_{i}} \frac{D(s)}{\rho(s)} d s
$$

In a ring the term $R_{56}(s)$ is a function of the azimuth, and is obtained by applying Eq. (2).

In a storage ring for ultrarelativistic particles above the transition energy the momentum compaction $\alpha_{c}$, which describes the correlation between the energy deviation and the trajectory length, is given by the integral of the dispersion function $D(s)$ divided by the bending radius $\rho(s)$ along the dipoles:

$$
\alpha_{c}=\frac{\Delta L}{L} / \frac{\Delta p}{p}=\frac{1}{L} \int \frac{D}{\rho} d s=\frac{1}{L} \sum_{i=1}^{n_{D}} \int_{0}^{l_{i}} \frac{D(s)}{\rho(s)} d s
$$

being $n_{D}$ the total number of dipoles and $l_{i}$ the $i$ th dipole magnetic length. Usually with the convention of $\rho(s)$ positive for bending inward the ring center, $\alpha_{c}$ is positive, corresponding to longer trajectories for higher energy particles.

There is a similarity between Eqs. (3) and (4), and for all the positions $s$ in which $D(s)=D^{\prime}(s)=0$ holds

$$
R_{56}(s)=-\alpha_{c} L
$$

if the effect of the rf cavity is neglected.

Particles reach the rf cavity position $s_{\text {rf }}$ with a correlation in the longitudinal phase plane which is defined by the value of $R_{56}\left(s_{\mathrm{rf}}\right)$. If the ring is isochronous the momentum compaction is zero and so is the total ring longitudinal phase advance. Usually this condition corresponds to $R_{56}\left(s_{\mathrm{rf}}\right)=0$, which means that all particles, regardless their energy, reach the cavity at the same instant and the mechanism of the synchrotron motion is not risen.

Let us now revert to the description of the only longitudinal plane, and following the formalism of Ref. [3] we describe the longitudinal oscillation in a $2 \times 2$ formalism, where the longitudinal plane is defined by the vector $(\delta l, \delta E / E)$. In this approximation we are neglecting the contribution to the bunch longitudinal dimensions of the horizontal bunch dimensions, which in fact increases the equilibrium bunch dimensions in the zones of the ring with dispersion, but this will be the subject of a different paper.

The only elements affecting the longitudinal single particle dynamics are $\mathrm{rf}$ cavities and bending magnets. The effect of an rf cavity, with rf wavelength $\lambda_{\mathrm{rf}}$, on the bunch with energy $E$ is described by the thin lens focusing matrix:

$$
\mathbf{M}_{\mathrm{rf}}=\left(\begin{array}{ccc}
1 & \frac{2 \pi}{E / e} \frac{V_{\mathrm{rf}}}{\lambda_{\mathrm{rf}}} & 1
\end{array}\right)=\left(\begin{array}{cc}
1 & 0 \\
-U & 1
\end{array}\right),
$$

where $U$ represents the voltage $V_{\text {rf }}$ derivative at the synchronous phase. The effect of a zone with dipoles between $s_{1}$ and $s_{2}$ is described by the "drift" matrix:

$$
\mathbf{M}\left(s_{2}-s_{1}\right)=\left(\begin{array}{cc}
1 & \int_{s_{1}}^{s_{2}} \frac{D\left(s^{\prime}\right)}{\rho\left(s^{\prime}\right)} d s^{\prime} \\
0 & 1
\end{array}\right) .
$$

In a ring with one cavity placed at $s_{\text {rf }}$, (see Fig. 1$)$ the oneturn matrix at the point $s$ is given by

$$
\mathbf{M}(s)=\mathbf{M}\left(s-s_{\mathrm{rf}}\right) \mathbf{M}_{\mathrm{rf}} \mathbf{M}\left(s_{\mathrm{rf}}-s\right) .
$$

Let us define the drift functions

$$
R_{1}(s)=\int_{s}^{s_{\mathrm{rf}}} \frac{D\left(s^{\prime}\right)}{\rho\left(s^{\prime}\right)} d s^{\prime} \quad \text { and } \quad R_{2}(s)=\int_{s_{\mathrm{rf}}}^{s} \frac{D\left(s^{\prime}\right)}{\rho\left(s^{\prime}\right)} d s^{\prime}
$$

computed in the clockwise direction along the ring, representing the drift of the longitudinal phase plane ellipse, i.e., the relative longitudinal position change between the cavity and the point $s$ of a particle with respect to the synchronous one in terms of its energy deviation $\delta E / E$. The drift functions are related to the momentum compaction by

$$
R_{1}(s)+R_{2}(s)=\alpha_{c} L
$$

The one-turn transport matrix (8) becomes

$$
\begin{aligned}
\mathbf{M}(s) & =\left(\begin{array}{cc}
1-U R_{2}(s) & \alpha_{c} L-U R_{1}(s) R_{2}(s) \\
-U & 1-U R_{1}(s)
\end{array}\right) \\
& =\cos \mu \mathbf{I}+\sin \mu\left(\begin{array}{cc}
\alpha_{L}(s) & \beta_{L}(s) \\
-\gamma & -\alpha_{L}(s) \cos
\end{array}\right)
\end{aligned}
$$

and from it the longitudinal Twiss parameters can be written as

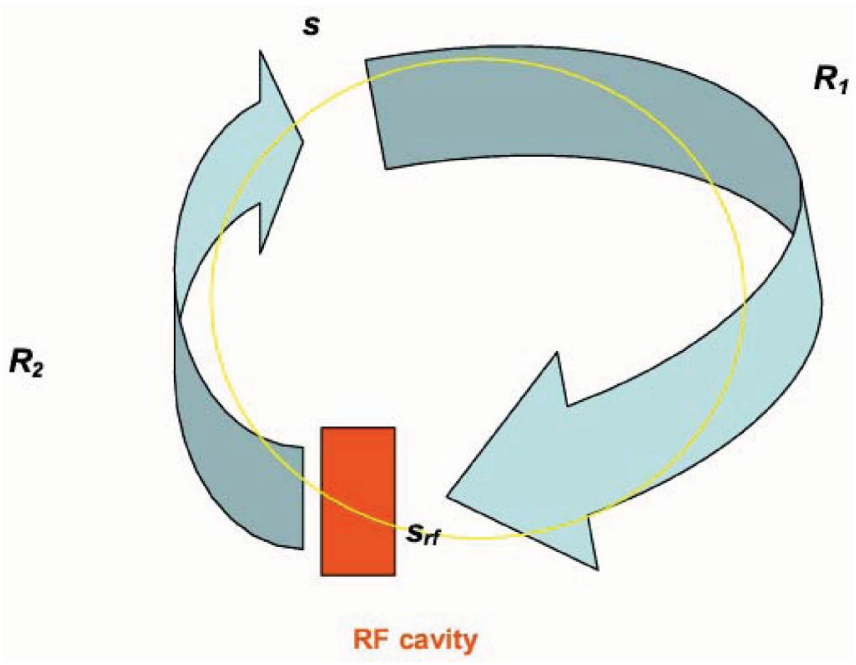

FIG. 1. (Color) Sketch of a ring with one cavity. 
$\cos \mu=1-\frac{\alpha_{c} L}{2} U$

$\beta_{L}(s)=\frac{1}{\sin \mu}\left[\alpha_{C} L-R_{1}(s) R_{2}(s) U\right], \quad \gamma_{L}=\frac{U}{\sin \mu}$.

The longitudinal beta function oscillates along the ring if the $U$ parameter is large and/or the drift functions are not negligible. It is clear from the expression of $\cos \mu$ that the sign of $U$ must be the same of $\alpha_{c}$ and for stable motion must be

$$
U_{\alpha_{C}} L<4
$$

as reported in [3]. Furthermore for positive (negative) $\alpha_{c}$, $\sin \mu$ will be positive (negative).

In order to illustrate the behavior of the longitudinal bunch modulation we use an example, based on parameters similar to DAФNE ones: let us consider a ring $100 \mathrm{~m}$ long, with energy $510 \mathrm{MeV}$, and an rf system at $1.3 \mathrm{GHz}$, as proposed for the DA $\Phi$ NE experiment on strong rf focusing [6].

The longitudinal phase advance is defined by the momentum compaction and the rf voltage, as shown in Fig. 2, where different curves correspond to different values of $\alpha_{c}$ for $\mathrm{rf}$ voltages in the range 1 to $10 \mathrm{MV}$. The beta function modulation is defined by $\alpha_{c}, V_{\mathrm{rf}}$, and $R_{1}(s)$. For weak rf focusing ( $U$ small), $\beta_{L}$ can be approximated by

$$
\beta_{L} \rightarrow \frac{\alpha_{C} L}{\sin \mu}
$$

and this is the case for most storage rings.

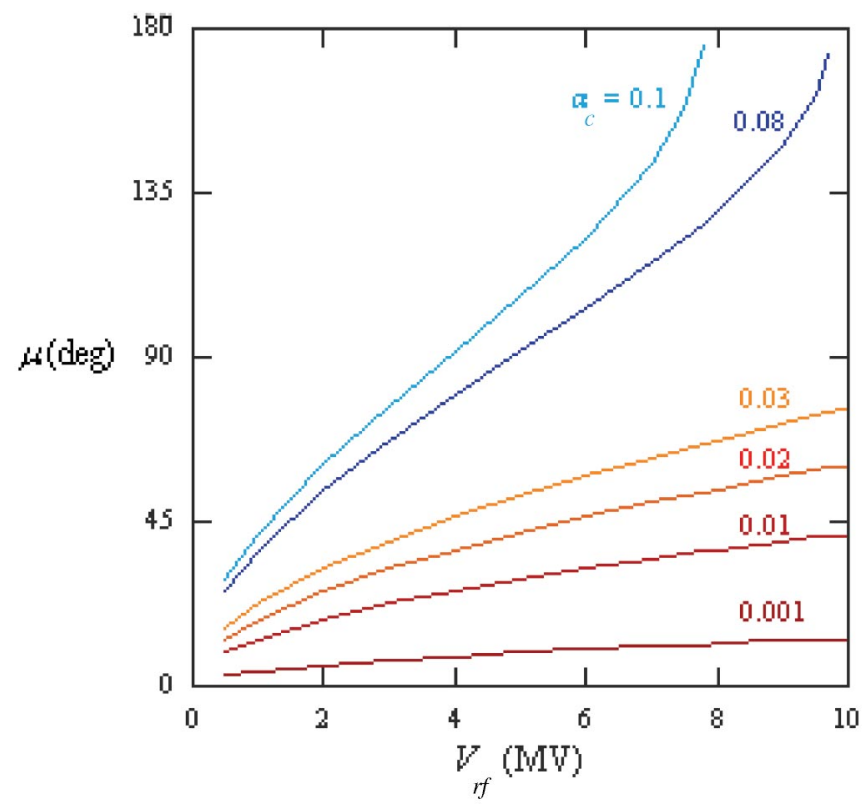

FIG. 2. (Color) Longitudinal phase advance as a function of the rf voltage at $1.3 \mathrm{GHz}, E=510 \mathrm{MeV}$ for different values of $\alpha_{c}$.
When $\beta_{L}(s)$ is not constant its minimum $\beta_{L \text { min }}$ appears at

$$
\frac{\partial \beta_{L}}{\partial s}=0 \Rightarrow R_{1}\left(s_{\min }\right)=\frac{\alpha_{c} L}{2},
$$

and is equal to

$$
\beta_{L \min }=\frac{\alpha_{c} L}{2 \sin \mu}(1+\cos \mu)
$$

which can be written as a function of the rf lens and momentum compaction:

$$
\beta_{L \min }=\sqrt{\frac{\alpha_{c} L\left(4-\alpha_{c} L U\right)}{4 U}}
$$

Figure 3 shows the behavior of the minimum beta as a function of the momentum compaction for different values of the rf voltage for our reference ring. It is clear from the figure the limit of stability for the high synchrotron tune region, corresponding to Eq. (13).

Let us now consider two cases:

A: the functions $R_{1}(s)$ and consequently $R_{2}(s)$ are monotonic in $s$.

$\mathrm{B}: R_{1}(s)$ is not monotonic, but has positive derivative in one part of the ring and negative in the other one.

\section{A. $R_{1}(s)$ monotonic}

The strong rf focusing principle corresponds to this case. The description of the bunch length behavior along the ring can be found in reference [3]. Here some of the principal characteristics will be reported.

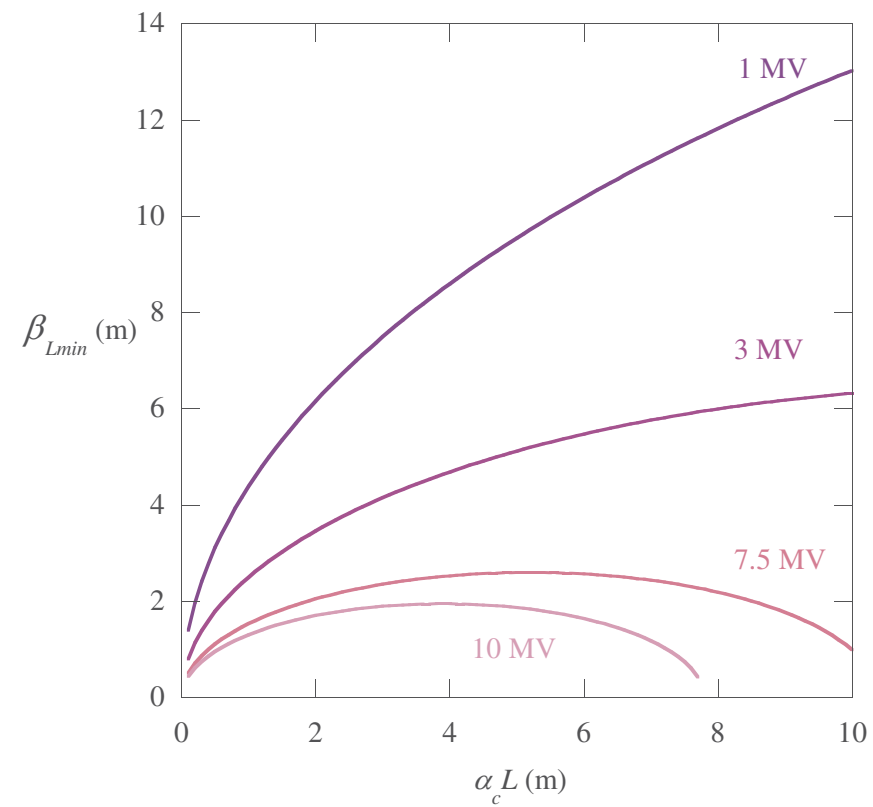

FIG. 3. (Color) Minimum longitudinal betatron function for different values of the rf voltage as a function of the momentum compaction. 
Let us consider a simplified case with linear behavior of $R_{1}(s)$ as shown in Fig. 4:

$$
R_{1}(s)=\alpha_{c} s, \quad R_{2}(s)=\alpha_{c}(L-s)
$$

for different values of $V_{\mathrm{rf}}(3,7.5 \mathrm{MV})$ and $\alpha_{c}$ (0.08 and 0.1 ). The corresponding longitudinal beta functions are reported in Fig. 5. Full lines correspond to the high value of $V_{\text {rf }}$, dashed ones to the lower value. The maximum of the function corresponds to the cavity position $\left(s_{\mathrm{rf}}=0\right)$, while the minimum is on the opposite side, where the function $R_{1}(s) R_{2}(s)$ has its maximum and the condition (15) is satisfied. Defining the modulation factor $F_{m}$ as the ratio between the maximum and the minimum value of the bunch length $\left[F_{m}=\operatorname{sqrt}\left(\beta_{L \text { max }} / \beta_{L \text { min }}\right)=\right.$ $\left.\sigma_{L \max } / \sigma_{L \min }\right]$, it can be observed that a noticeable value of $F_{m}$ appears only at high voltage and high $\alpha_{c}$, when the longitudinal phase advance $\mu$ approaches $180^{\circ}$.

If $R_{1}(s)$ is not linear the form of the betatron function will not be exactly parabolic [see Eq. (12)]. Consider also that in a real ring both functions $R_{1}(s)$ and $\beta_{L}(s)$ are step functions since they change only inside the dipoles, while they are constant elsewhere.

\section{B. $R_{1}(s)$ nonmonotonic}

Let us now consider the case in which $R_{1}(s)$ is not monotonic, having positive derivative in one part of the ring and negative in the other one, which is possible in the case that in one part of the ring the dispersion in dipoles is always positive, while in the second part of the ring there is predominance of negative dispersion in dipoles. The momentum compaction will be defined by the value of $R_{1}(L)$ and it can go to very small values, up to the limit of isochronicity.

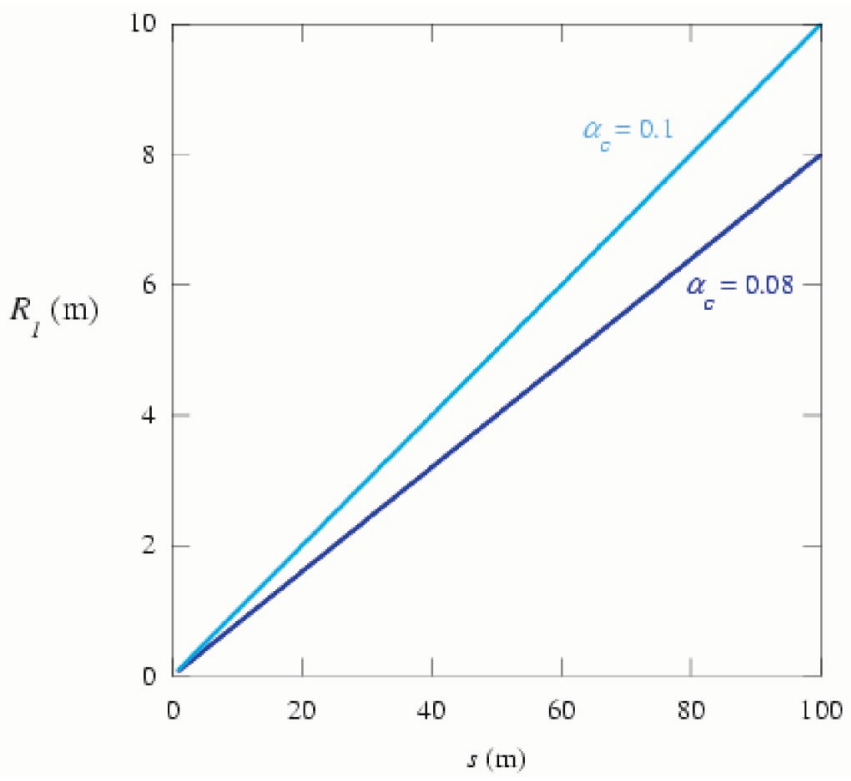

FIG. 4. (Color) Function $R_{1}(s)$ along the ring for $\alpha_{c}=0.08,0.1$.

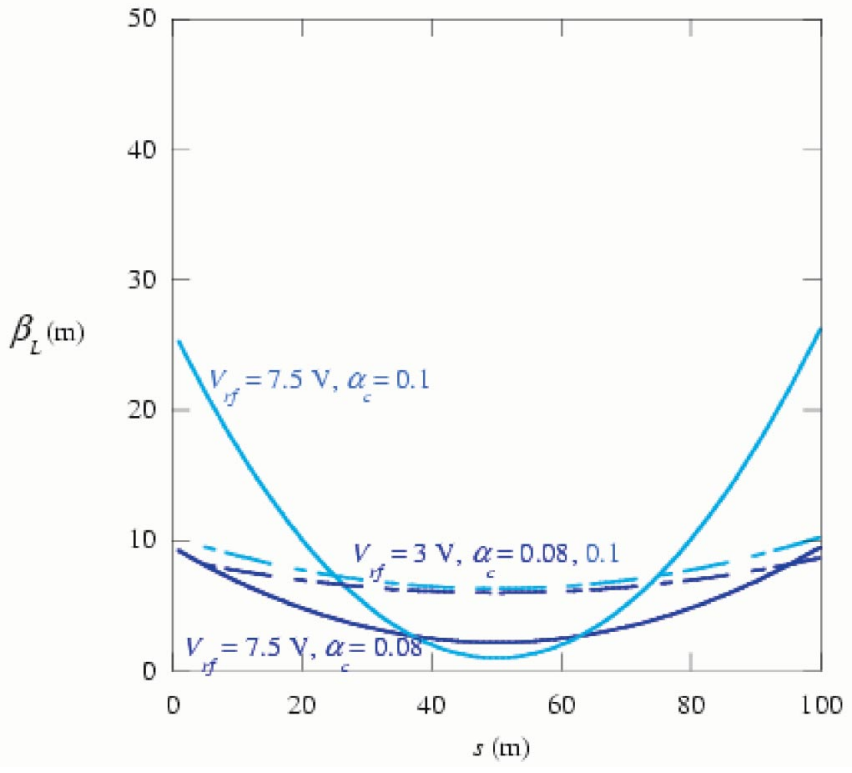

FIG. 5. (Color) Longitudinal beta function along the ring for $\alpha_{c}=0.08$ and 0.1 in the monotonic case for two values of $V_{\mathrm{rf}}$ (3 and 7.5 MV). Darker color corresponds to lower $a_{c}$.

The isochronicity is usually obtained by a minimization of the integral appearing in Eq. (4) along the ring, but it can be also a result of balancing the positive and the negative contribution to this integral so that the behavior of the $R_{56}(s)$ term along the ring oscillates. This means that the longitudinal betatron function will be modulated along the ring or, equivalently, looking at the longitudinal phase space in different positions along the ring, the ellipse rotates and there is a correlation between energy deviation and position in the bunch. The rotation is clockwise (counterclockwise) for positive (negative) contributions to the integral (4).

A position where the term $R_{56}(s)$ of the one-turn matrix $\mathbf{R}(s)$ is high, and so is the longitudinal beta function, corresponds to a position in which the longitudinal phase space is highly correlated, thus meaning that particles with different energies reach that point at different times, even if the ring is isochronous, analogously to what happens in a nonzero momentum compaction ring. Particles with different energies follow equal length trajectories, but they are ahead or after the synchronous one depending in which part of the ring they are traveling.

In principle it could be thought that placing the rf cavity in a position in which the energy-position correlation is high it could be possible to give rise to the synchrotron oscillations and make the isochronous ring stable. This is not true since the rf cavity itself changes the longitudinal phase advance behavior along the ring, as will be shown in the following.

Let us consider the same ring of case A with the same behavior of $R_{1}(s)$ in half the ring, while in the second part of the ring $R_{1}(s)$ is a linear function with negative deriva- 


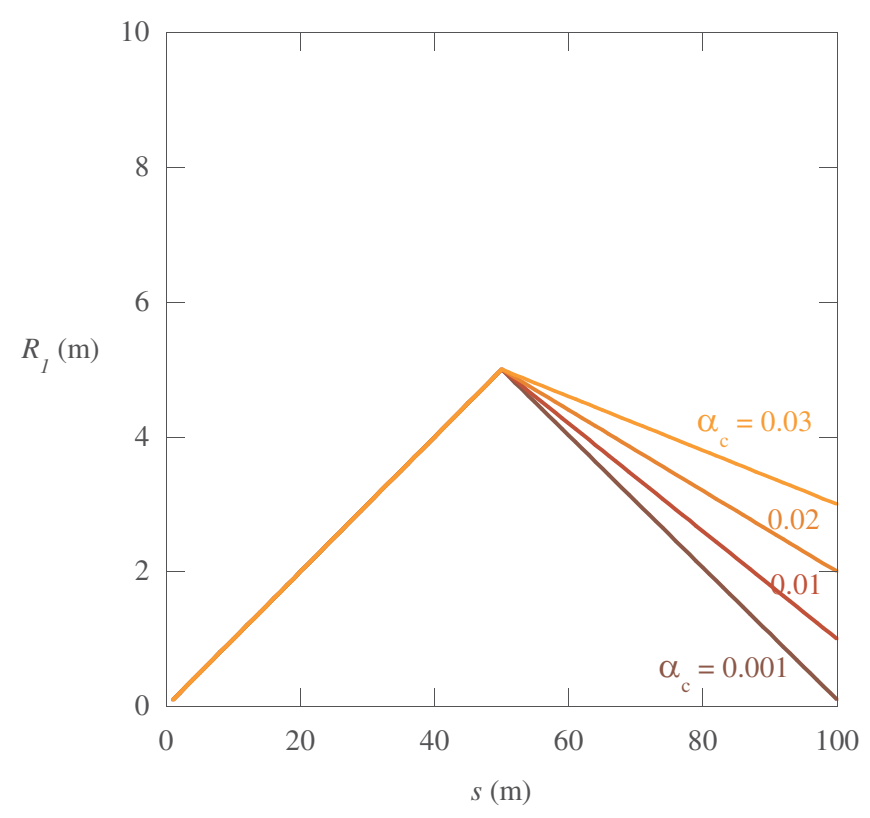

FIG. 6. (Color) Function $R_{1}(s)$ along the ring in case $\mathrm{B}$, for four different values of $\alpha_{c}(0.001,001,0.02,0.03$, from darker to lighter colors).

tive. Changing the derivative value, different values of $\alpha_{c}$ are obtained. Figure 6 shows the $R_{1}(s)$ behavior in the ring for four different values of $\alpha_{c}(0.001,0.01,0.02,0.03$ - the darker colors correspond to smaller $\alpha_{c}$ ). Let us consider that the cavity is placed in a point where $R_{1}(s)$ changes the slope sign, in particular, at $s=0$. Figure 7 shows the

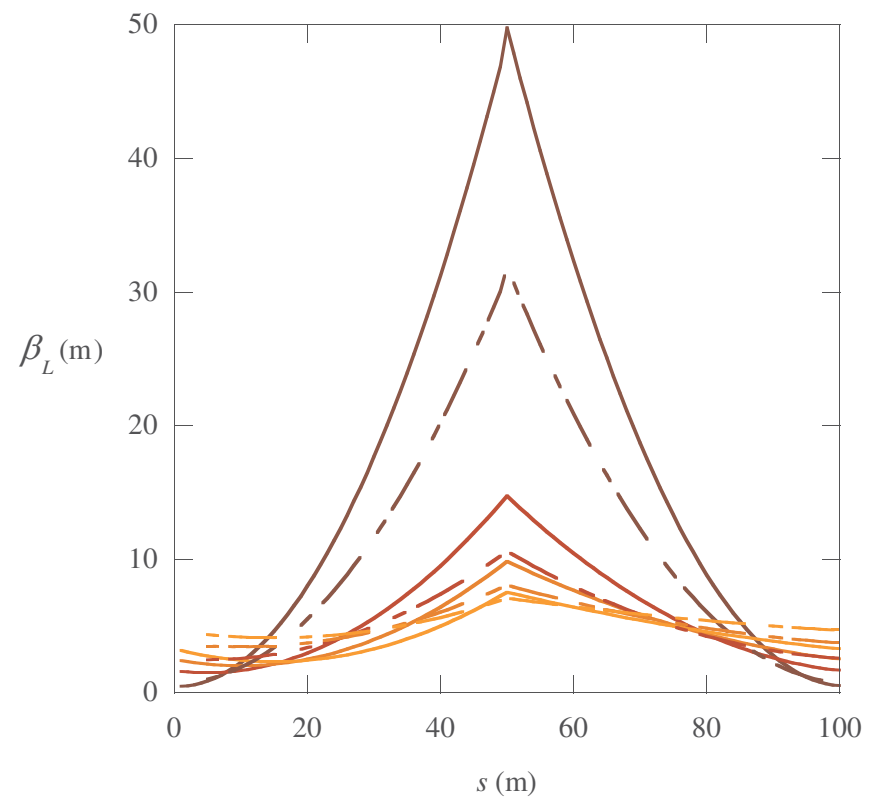

FIG. 7. (Color) Longitudinal beta function for case B. Full lines correspond to $V_{\mathrm{rf}}=7.5 \mathrm{MV}$, dashed ones to $V_{\mathrm{rf}}=3 \mathrm{MV}$. The highest modulation corresponds to the smaller value of $\alpha_{c}$, and the different colors to the four values of $\alpha_{c}$, as in the previous figure. corresponding $\beta_{L}$ behavior for $V_{\mathrm{rf}}=3 \mathrm{MV}$ (dashed lines) and $V_{\mathrm{rf}}=7.5 \mathrm{MV}$ (full lines). The scales are the same of Fig. 5. The asymptotic limit for the maximum modulation factor $F_{m}$ corresponds to the isochronicity condition. To be noticed that in this case the minimum corresponds to the cavity position for the isochronous case and it moves to the point in which the function $R_{1}(s) R_{2}(s)$ is maximum, which is near the cavity if $\alpha_{c}$ is small, as can be simply deduced by Eq. (15). The maximum corresponds to the point where the function $R_{1}(s)$ changes the sign of its derivative and this is a difference with respect to the case of monotonic behavior of $R_{1}(s)$.

If the cavity is placed in a point where the $R_{1}(s)$ derivative has constant sign, two minima appear, since there are two points in the ring satisfying Eq. (15), and their value is the same as defined by Eq. (16). $F_{m}$ decreases as the distance of the cavity from the point where $d R_{1}(s) / d s$ changes sign increases. Figure 8 shows this effect for the quasi isochronous case $\left(\alpha_{c}=0.001\right)$ as the cavity moves from the initial point to the central point of the ring.

\section{ENERGY SPREAD AND LONGITUDINAL EMITTANCE}

The natural longitudinal emittance and energy spread usually refer to the approximation of small synchrotron tune and small derivative of $R_{1}(s)$. The energy spread is defined by the eigenvalues of the matrix $\mathbf{M}(s)$ [7], considering radiation damping and energy emission. The expressions for the strong rf focusing case are given in [3], where the formalism implies that the cavity is placed at $s=0$.

They can be rewritten in the more general formalism, using the same approach, and we obtain for the energy spread

$$
\left(\frac{\sigma_{E}}{E}\right)^{2}=\frac{\gamma_{L}}{2} C_{L} \frac{\gamma^{5}}{L \alpha_{\|}} \int \frac{\beta_{L}(s)}{|\rho(s)|^{3}} d s,
$$

with $C_{L}=2.15 \times 10^{-19} \mathrm{~m}^{3} \mathrm{sec}^{-1}, \alpha_{\|}$the longitudinal damping factor, defined by

$$
\alpha_{\|}=\frac{C_{\alpha} E^{3}}{L}\left(2 I_{2}+I_{4}\right)
$$

with $C_{\alpha}=2113.1 \mathrm{~m}^{2} / \mathrm{GeV}^{3} / \mathrm{s}$ and $I_{j}$ the usual synchrotron radiation integrals [5].

Remembering that in the limit of small synchrotron oscillations the energy spread is given by [5]:

$$
\left(\frac{\sigma_{E}}{E}\right)_{0}^{2}=\frac{C_{L}}{2} \frac{\gamma^{5}}{L \alpha_{\|}} I_{3}
$$

we can write that in the bunch length modulation regime the energy spread is increased by the factor $G\left(U, \alpha_{c} L\right)$ [3]:

$$
\left(\frac{\sigma_{E}}{E}\right)^{2}=\left(\frac{\sigma_{E}}{E}\right)_{0}^{2} G\left(U, \alpha_{c} L\right)=\left(\frac{\sigma_{E}}{E}\right)_{0}^{2} \frac{\gamma_{L}}{I_{3}} \int \frac{\beta_{L}(s)}{|\rho(s)|^{3}} d s,
$$

which for small amplitudes tends to the unity. 

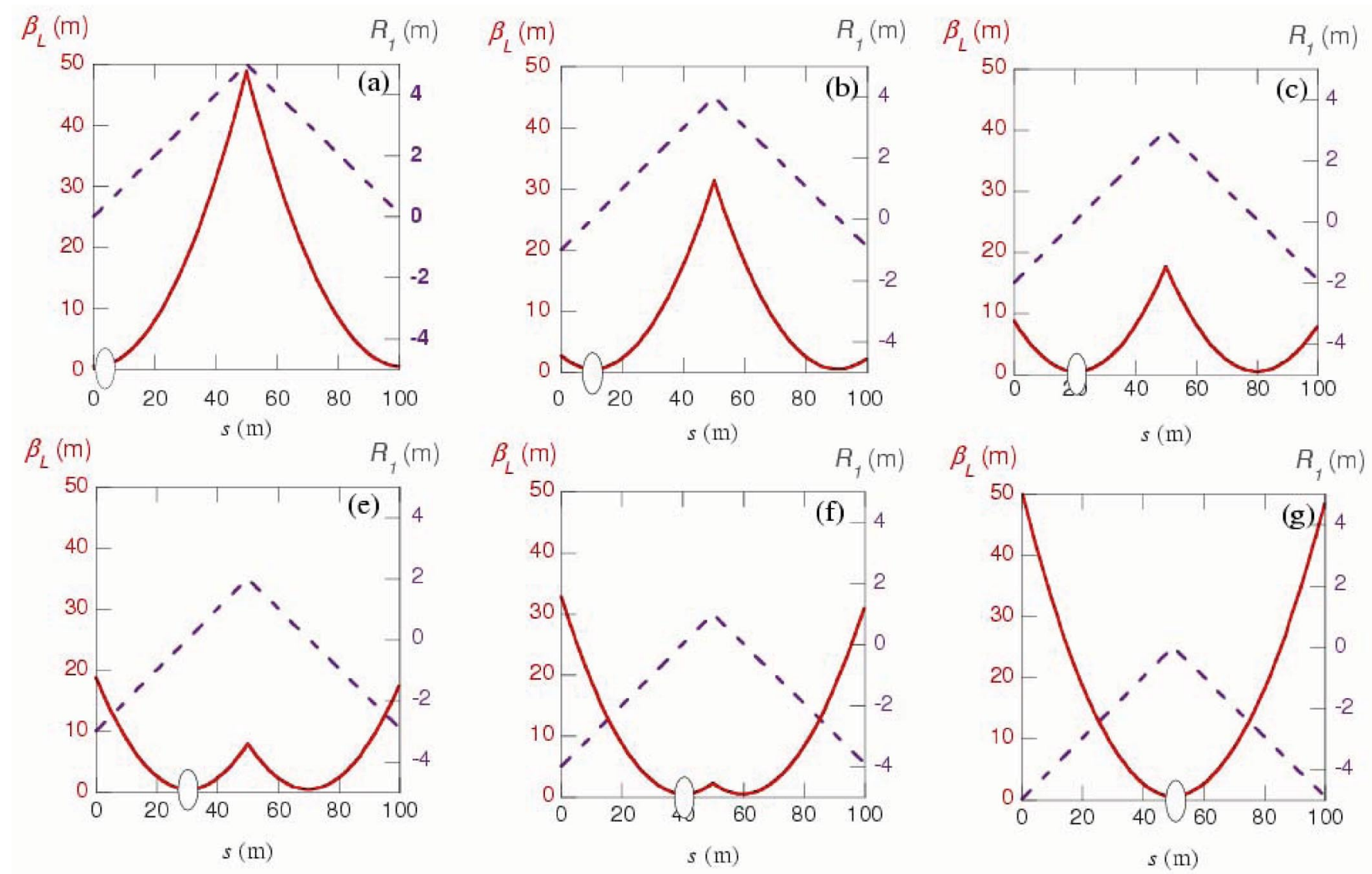

FIG. 8. (Color) $\beta_{L}$ (full red lines) and $R_{1}(s)$ (black dashed lines) along the ring for different positions of the cavity for a quasi isochronous ring $\left(\alpha_{c}=0.001\right)$ in the case B for $V_{\mathrm{rf}}=7.5 \mathrm{MV}$.

The longitudinal emittance is [3]:

$$
\varepsilon_{L}=\frac{1}{\gamma_{L}}\left(\frac{\sigma_{E}}{E}\right)^{2}
$$

It is interesting to compare the case of A high synchrotron tune $Q_{s}=\mu / 2 \pi$-monotonic behavior of $R_{1}(s)$ against the case of B low synchrotron tune-nonmonotonic $R_{1}(s)$.

There is an increase both in emittance and in energy spread as $F_{m}$ is increased. In case $\mathrm{A}$ it comes from the approaching of $\mu$ to $180^{\circ}$, in case B the modulation can be increased by decreasing $\alpha_{c}$ and therefore $\mu$ tends to zero. In the high $Q_{s}$ regime given a certain lattice the maximum voltage is limited by expression (13), while in the low $Q_{s}$ regime the modulation factor can be increased for a certain $\alpha_{c}$ in a much larger range, even if of course the emittance will grow according to expressions (19) and (23).

Let us refer again to our reference ring described before. Let us assume that the damping time is the same in all the cases, even if this is not completely true due to the dependence of $\alpha_{\|}$on the synchrotron radiation integral $I_{4}$, which depends on the dispersion function. We can assume that $I_{4}$ is zero, for example, in the case in which the dipoles in the ring are sector magnets with field index $n=0.5$. A reasonable damping time is of the order of $20 \mathrm{msec}$, and with $\rho=1 \mathrm{~m}$, constant in the whole ring we can compute the energy spread for all the cases (a) and (b) described above.

The emittance and the energy spread are plotted, respectively, in Figs. 9(a) and 9(b) for $V_{\mathrm{rf}}=3$ and 7.5 MV in all cases cited above.

In all cases the larger $F_{m}$, the larger the beam energy spread and emittance. The same $F_{m}$ is obtained in the nonmonotonic behavior with lower if voltage than in the monotonic case if the value of $\alpha_{c}$ is small. Both regimes have advantages and drawbacks: the high $\alpha_{c}$ increases the microwave instability threshold, while the high $Q_{s}$ crowds with strong resonances the working point footprint. In the monotonic case there is the advantage of placing the $\mathrm{rf}$ cavity in the zone where the bunch is longer and therefore less sensitive to the cavity impedance.

\section{BUNCH LENGTH}

The bunch length along the ring is given by

$$
\sigma_{L}(s)=\sqrt{\varepsilon_{L} \beta_{L}(s)}
$$

and again let us remember that the influence of transverse dynamics on the bunch dimensions through the terms $R_{51}$, $R_{52}$ of the $6 \times 6$ matrix are not taken into account. A complete treatment needs to add also these terms, because 

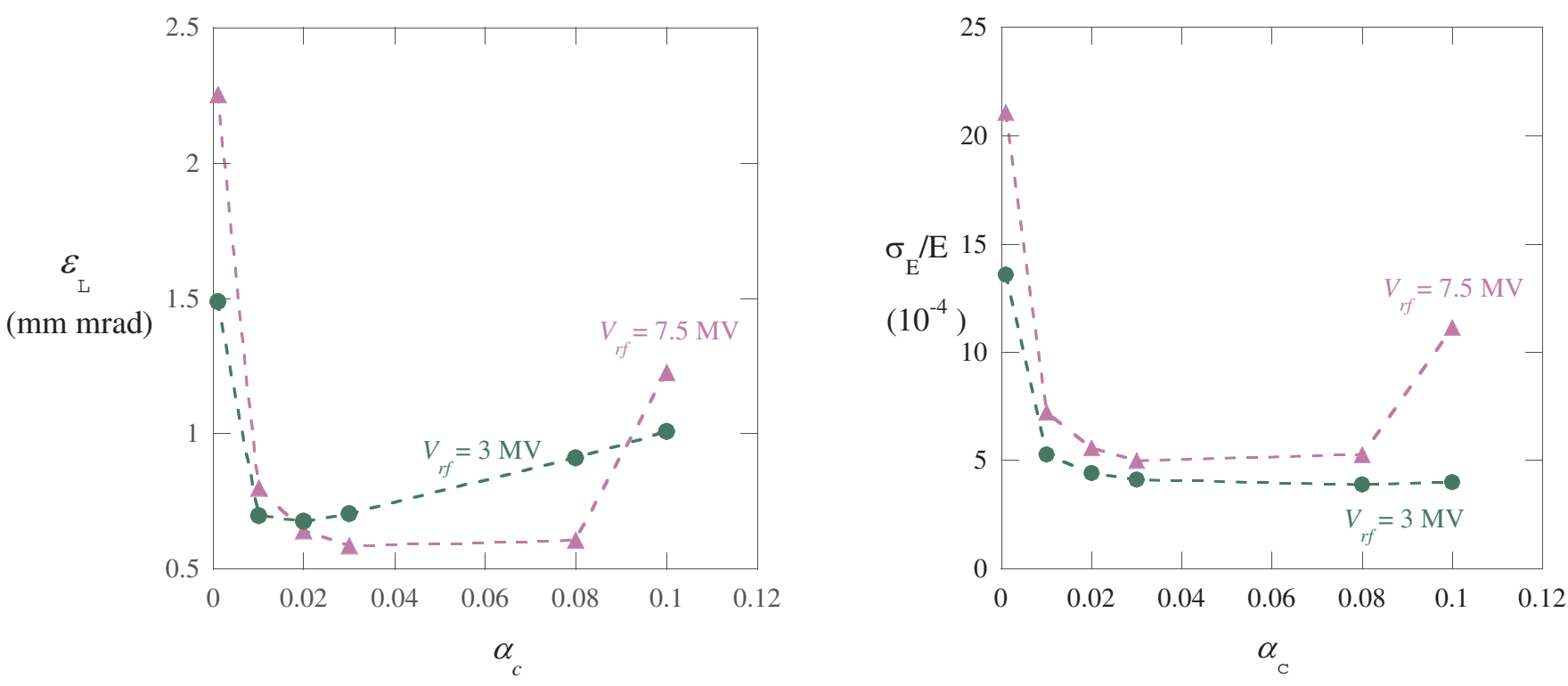

FIG. 9. (Color) Longitudinal emittance (a) and energy spread (b) for the reference ring at two different value of $V_{\text {rf }}$. Dots correspond to $V_{\mathrm{rf}}=3 \mathrm{MV}$, triangles to $V_{\mathrm{rf}}=7.5 \mathrm{MV} . R_{1}(s)$ is nonmonotonic for the first four points, monotonic for the two last ones.

they are not negligible since they growth with the dispersion function, which is large for such regimes. Anyway in the points where the minimum bunch length is required these effects are negligible, if we remain in the linear approximation.

When the synchrotron tune approaches zero or the half integer, the minimum $\beta_{L}$ function goes to zero [see Eq. (16) and Fig. 3], while the longitudinal emittance diverges, as also does the energy spread. The increase in the emittance transfers essentially to the energy spread, while the product of the emittance and $\beta_{L \text { min }}$ is determined by the behavior of the function $R_{1}(s)$.

The minimum bunch length along the ring can be deduced from Eqs. (16) and (23):

$$
\begin{aligned}
\sigma_{L \min } & =\sqrt{\varepsilon_{L} \beta_{L \min }} \\
& =\left(\frac{\sigma_{E}}{E}\right)_{0} \sqrt{\frac{\alpha_{C} L}{U}-\frac{1}{I_{3}} \int \frac{R_{1}(s) R_{2}(s)}{|\rho|^{3}} d s} \\
& =\left(\frac{\sigma_{E}}{E}\right) \sqrt{f\left(\alpha_{C}, U\right)-g_{\text {Latt }}}
\end{aligned}
$$

The first term under the square root, $f$, depends both on the lattice via $\alpha_{c}$, and on the cavity via the $U$ parameter, while the second term $g_{\text {Latt }}$ depends strictly on the lattice.

In the case of monotonic $R_{1}(s), g_{\text {Latt }}$ is positive and the minimum bunch length is determined by the difference between the two terms. In the hypothesis of constant $\rho(s)$ and linear behavior of $R_{1}(s)$ [3]

$$
\sigma_{L \min }=\alpha_{c} L\left(\frac{\sigma_{E}}{E}\right)_{0} \sqrt{\frac{2+\cos \mu}{6(1-\cos \mu)}}=\alpha_{c} L\left(\frac{\sigma_{E}}{E}\right)_{0} h(\mu) .
$$

The $\sigma_{L \text { min }}$ is determined almost linearly by the value of the momentum compaction since for $\mu \rightarrow \pi$ the adimensional function $h(\mu)$ tends asymptotically to the value 0.28 .

In the case of nonmonotonic $R_{1}(s)$, the factor $g_{\text {Latt }}$ is negative and the contribution of both terms adds. The smaller the $\alpha_{c}$, the larger $g_{\text {Latt }}$. The minimum bunch length for very small $\alpha_{c}$ depends only on the behavior of $R_{1}(s)$. Figures 10 show the bunch length for the cases described above, and it can be seen that the minimum bunch length is almost the same for all the small $Q_{s}$ values.

\section{TWO CAVITIES IN THE RING}

Let us consider now the case in which there are two different rf systems in the ring with dipoles in between (see Fig. 11).

Let us call $s_{\mathrm{rf} 1}$ and $s_{\mathrm{rf} 2}$ the position of the two cavities defined, respectively, by the parameters $U_{1}$ and $U_{2}$ and define the drift functions:

$$
\begin{aligned}
& R_{1}(s)=\int_{s}^{s_{\mathrm{r} 1} 1} \frac{D\left(s^{\prime}\right)}{\rho\left(s^{\prime}\right)} d s^{\prime}, \\
& R_{2}(s)=\int_{s_{\mathrm{r} 2}}^{s} \frac{D\left(s^{\prime}\right)}{\rho\left(s^{\prime}\right)} d s, \text { and } R_{3}=\int_{s_{\mathrm{rf} 1}}^{s_{\mathrm{r} 2}} \frac{D\left(s^{\prime}\right)}{\rho\left(s^{\prime}\right)} d s,
\end{aligned}
$$

following the scheme of Fig. 11. The one-turn transport matrix expression is the product of the section matrices:

$$
\mathbf{M}(s)=\mathbf{M}\left(s-s_{\mathrm{rf} 2}\right) \mathbf{M}_{\mathrm{rf} 2} \mathbf{M}\left(s_{\mathrm{rf} 2}-s_{\mathrm{rf} 1}\right) \mathbf{M}_{\mathrm{rf} 1} \mathbf{M}\left(s_{\mathrm{rf} 1}-s\right) .
$$

By multiplying the above expressions and considering that

$$
R_{1}(s)+R_{2}(s)+R_{3}=\alpha_{c} L
$$

the longitudinal phase advance and Twiss functions are 

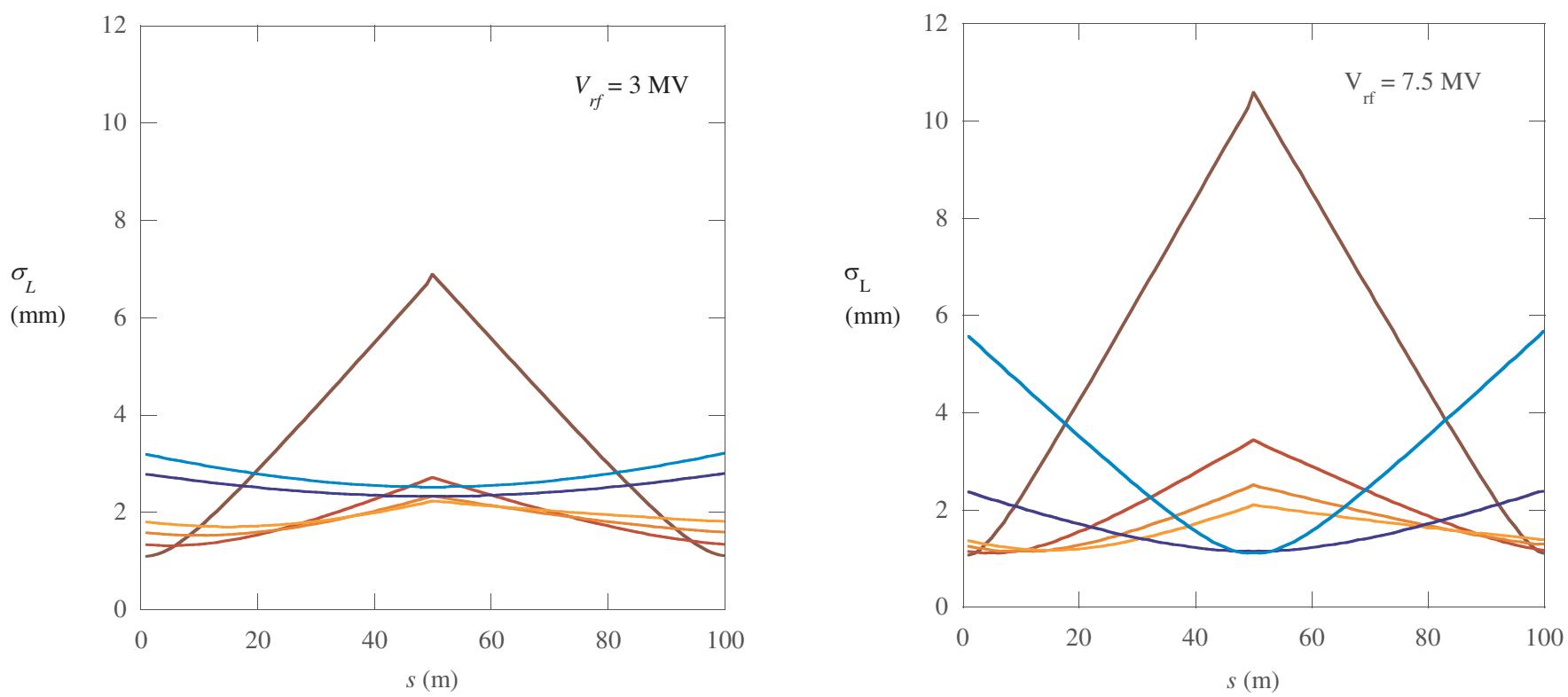

FIG. 10. (Color) Bunch length in $\mathrm{mm}$ along the ring for the example cited above. Blue lines, with the minimum at $s=50 \mathrm{~m}$, correspond to the monotonic case A and red to brown lines, with the maximum at $s=50 \mathrm{~m}$, to the nonmonotonic B. The cavity is placed at $s=0$.

given by

$$
\begin{aligned}
\cos \mu= & 1-\frac{\alpha_{c} L}{2}\left(U_{1}+U_{2}\right)+\left(\alpha_{c} L-R_{3}\right) R_{3} U_{1} U_{2}, \\
\beta_{L}(s)= & \frac{1}{\sin \mu}\left\{\alpha_{c} L-\left[R_{2}(s)+R_{3}\right] R_{1}(s) U_{1}-\left[R_{1}(s)\right.\right. \\
& \left.\left.+R_{3}\right] R_{2}(s) U_{2}+R_{1}(s) R_{2}(s) R_{3} U_{1} U_{2}\right\}, \\
\gamma_{L}= & \frac{1}{\sin \mu}\left(U_{1}+U_{2}-R_{3} U_{1} U_{2}\right) .
\end{aligned}
$$

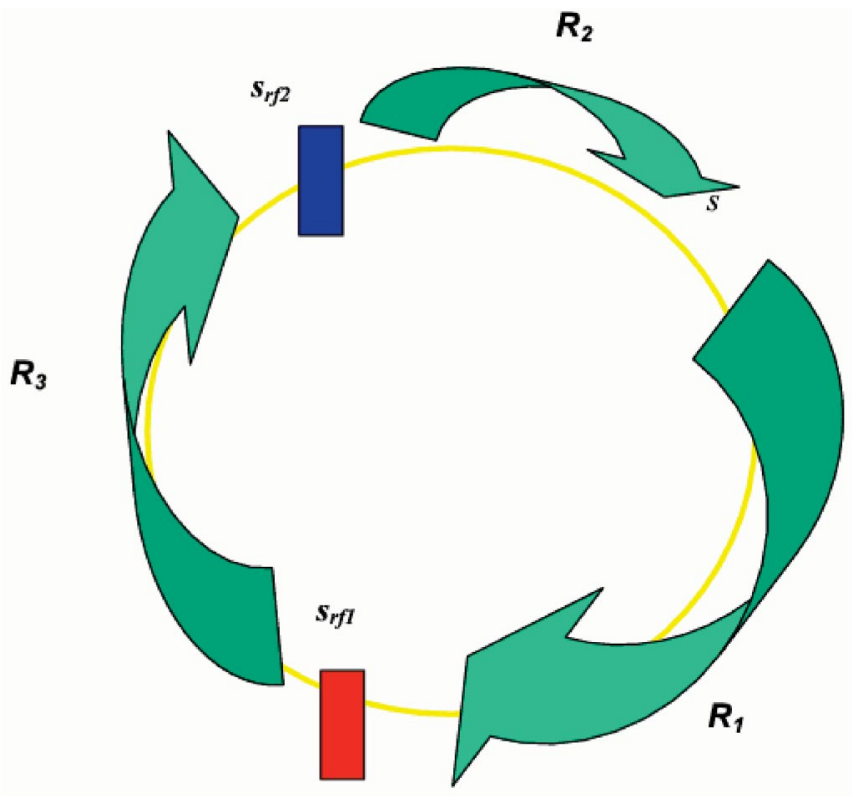

FIG. 11. (Color) Sketch of a two-cavities ring.
As expected the phase advance $\mu$ and the parameter $\gamma_{L}$ do not depend on the position along the ring, but do on the longitudinal drift between the rf cavities in terms of $R_{3}$. Notice that the expression of $\beta_{L}(s)$ is valid only for the arrangement of Fig. 11 in the zone between the first and the second cavity with clockwise rotation in the ring. To have the closed solution for $\beta_{L}$ in the zone between $s_{\mathrm{rf} 2}$ and $s_{\mathrm{rf} 1}$ the $R_{i}$ expressions must be accordingly modified.

From the above expressions it is clear that with multiple rf systems there are several knobs to act on the bunch length modulation, the phase advance and the longitudinal emittance. Without making a complete panorama on all the possible solutions, some considerations on few cases are reported as examples, again referred to our reference ring used before.

\section{A. Longitudinal phase advance $\mu$}

The value of the synchrotron tune once defined the rf systems depends only on the value of $R_{3}$ and $\alpha_{c}$ and not on the behavior of the functions $R_{1}(s)$ or $R_{2}(s)$. According to the distance between the rf cavities in terms of the drift $R_{3}$ parameter, the ring synchrotron tune in the case of high or low momentum compaction has different behavior. Figure 12 refers to our example ring, considering two equal rf systems $\left(U_{1}=U_{2}\right)$. When the two cavities are close in terms of $R_{3}$, the ring behaves in a similar way as in the case of the single rf system $\left(R_{3}=1 \mathrm{~m}\right.$ in the figures). When $R_{3}$ increases the value of $\mu$ can go to zero for the high momentum compaction case, while it increases for the small momentum compaction one. It is possible therefore to obtain low synchrotron tune with high momentum compaction and vice versa. 

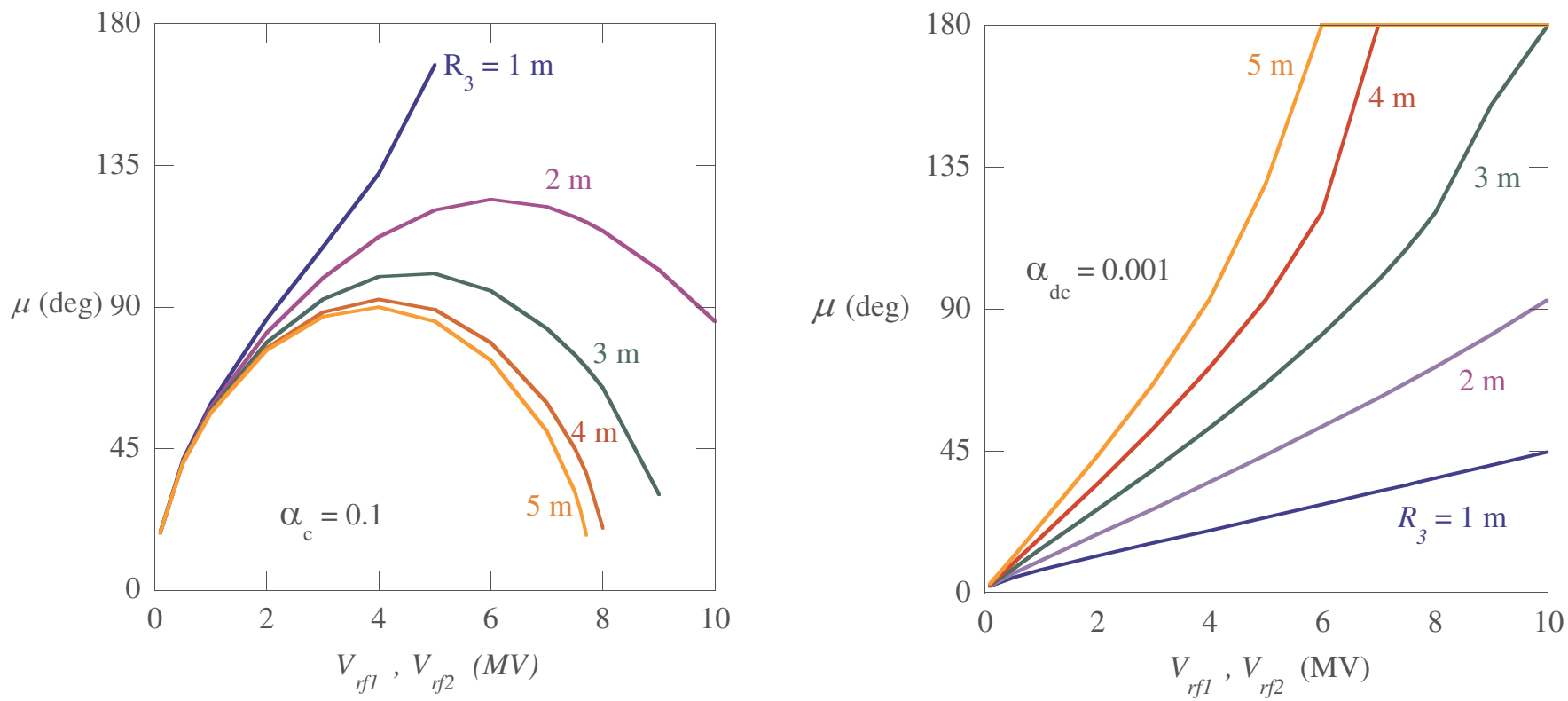

FIG. 12. (Color) Longitudinal phase advance as a function of $V$ in the hypothesis of equal cavities for different longitudinal drift between the cavities and for two different values of $a_{c}$.

Figure 13 shows the $\mu$ behavior with two different $\mathrm{rf}$ systems $\left(U_{1} \neq U_{2}\right)$ and high $a_{c}(=0.1)$ by varying $U_{2}$, keeping fixed $U_{1}$. The two cases correspond to $U_{1}$ small $\left(V_{\text {rf1 }}=2 \mathrm{MV}\right)$ and $U_{1}$ large $\left(V_{\text {rf1 }}=8 \mathrm{MV}\right)$. The vertical yellow lines show the common situation. When in the ring there is a weak cavity the presence of a second one increases the longitudinal phase advance, while if there is a strong cavity the second one decreases $\mu$.

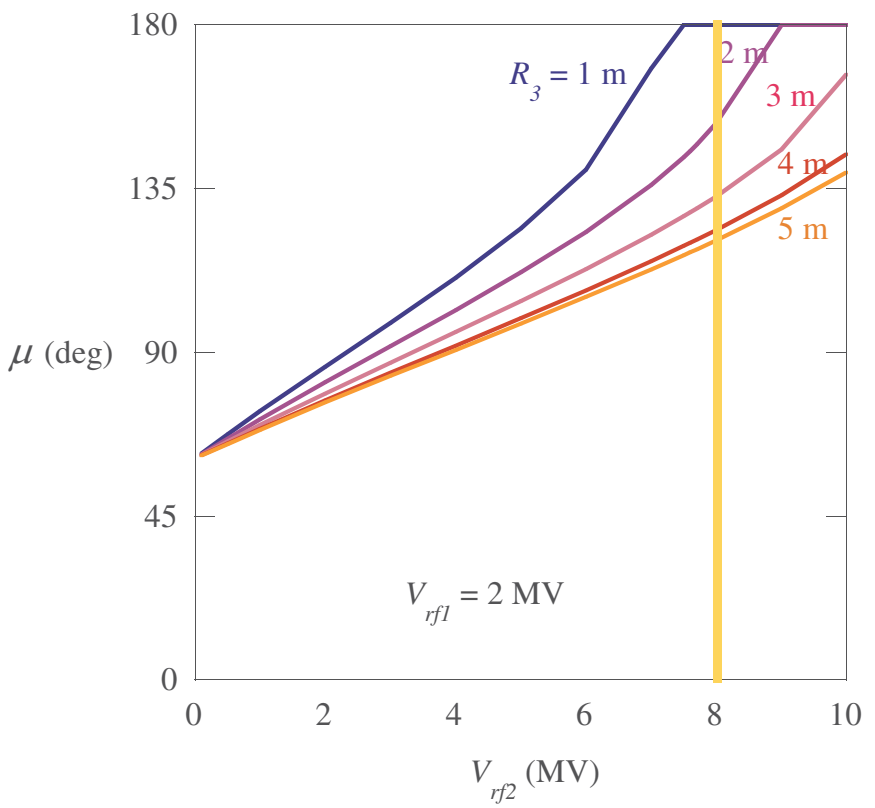

\section{B. Longitudinal beta function and bunch length}

The behavior of the $\beta_{L}$ function and the value of $\gamma_{L}$ as defined in Eq. (29) determine the energy spread and the emittance through expressions (19) and (23), which are valid also in the case of multiple rf systems along the ring.

Let us consider the monotonic ring with $\alpha_{c}=0.1$ and two equidistant cavities $\left(R_{3}=5 \mathrm{~m}\right)$ with different voltage $\left(U_{1} \neq U_{2}\right)$.

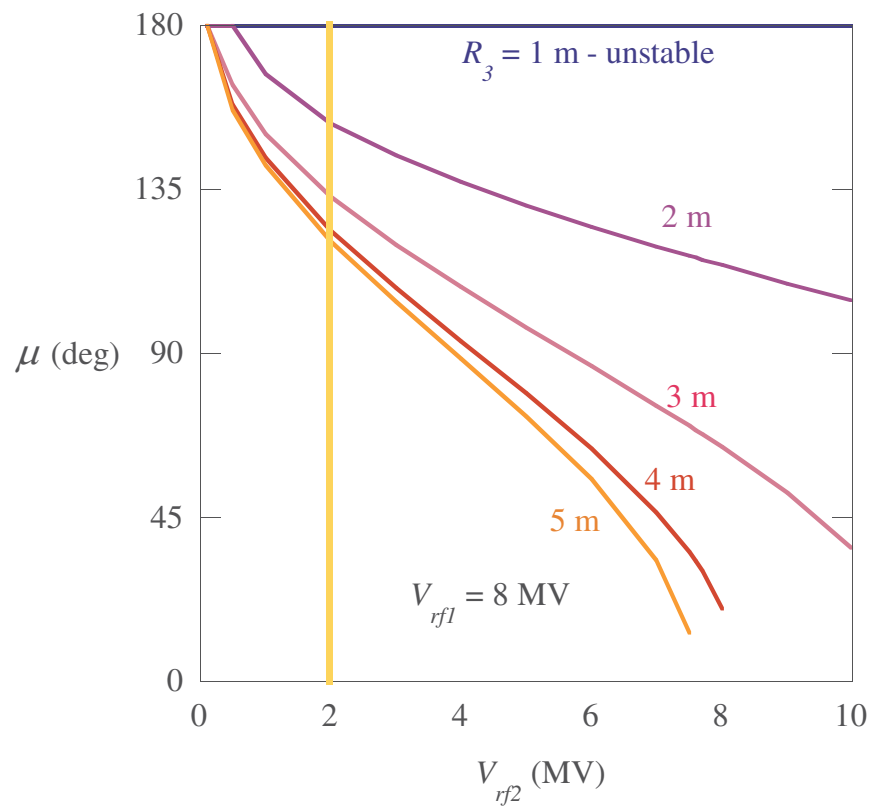

FIG. 13. (Color) Longitudinal phase advance as a function of the second rf cavity voltage in the case of the first weak rf cavity $\left(V_{\text {rf } 1}=2 \mathrm{MV}\right)$, or strong $\left(V_{\text {rf1 }}=8 \mathrm{MV}\right)$. The different curves correspond to different value of $R_{3}$. 

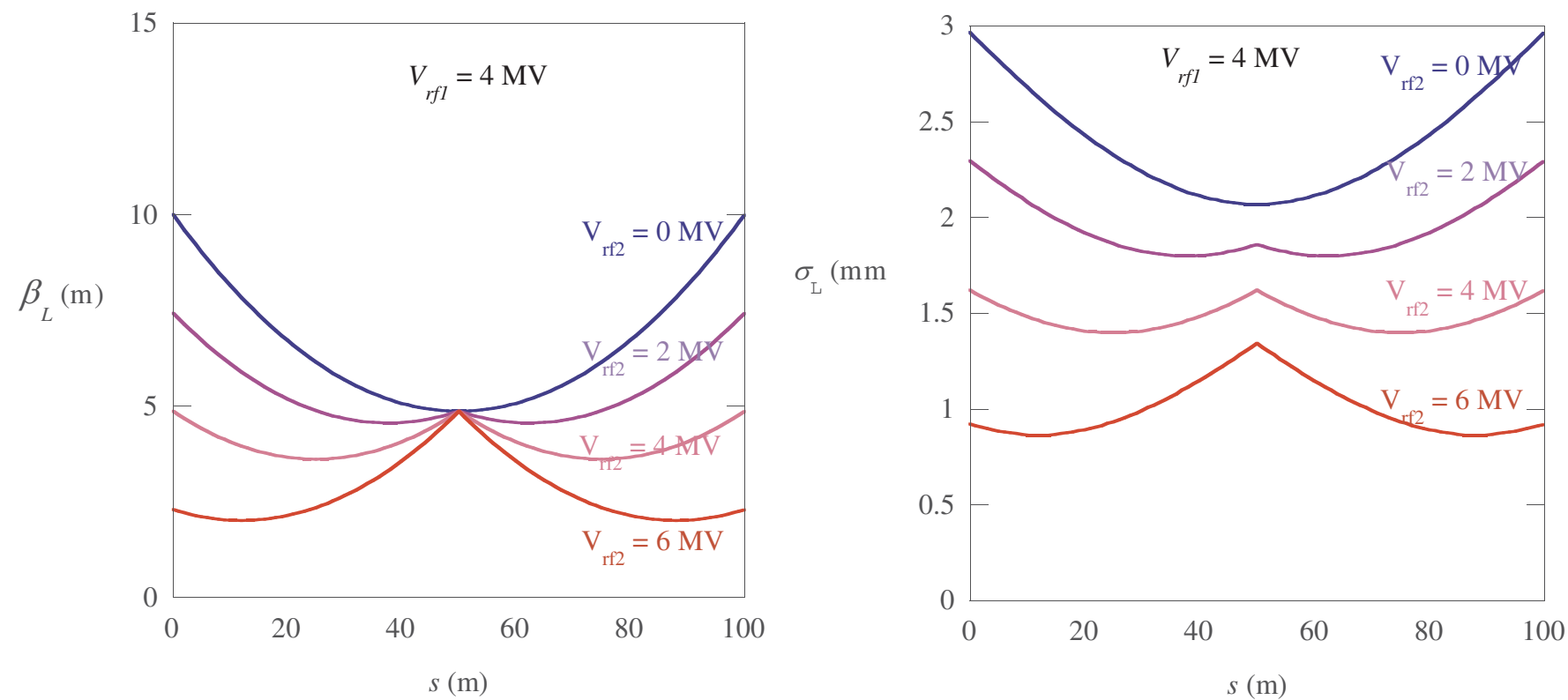

FIG. 14. (Color) Longitudinal beta function and bunch length along the ring for monotonic $R_{1}(s)$ with two cavities placed in symmetric points and different ratio of $U_{1} / U_{2}$.

Keeping the cavity at $s=0$ fixed $\left(V_{\mathrm{rf} 1}=4 \mathrm{MV}\right)$ and changing the voltage on the cavity placed at the opposite side of the ring, it is possible to move the position of the minimum bunch length along the ring, as shown in Fig. 14. The emittance and the energy spread decrease as $V_{\text {rf2 }}$ increases (see Fig. 15).

Interesting is also the solution in which the betatron function is almost constant and high in between the two cavities, having a minimum in one part of the ring, as shown in Fig. 16. In this case the two cavities are equal $\left(V_{\mathrm{rf} 1}=V_{\mathrm{rf} 2}=5 \mathrm{MV}\right)$ and the longitudinal drift in between is $R_{3}=3 \mathrm{~m}$. We are here considering a fictitious

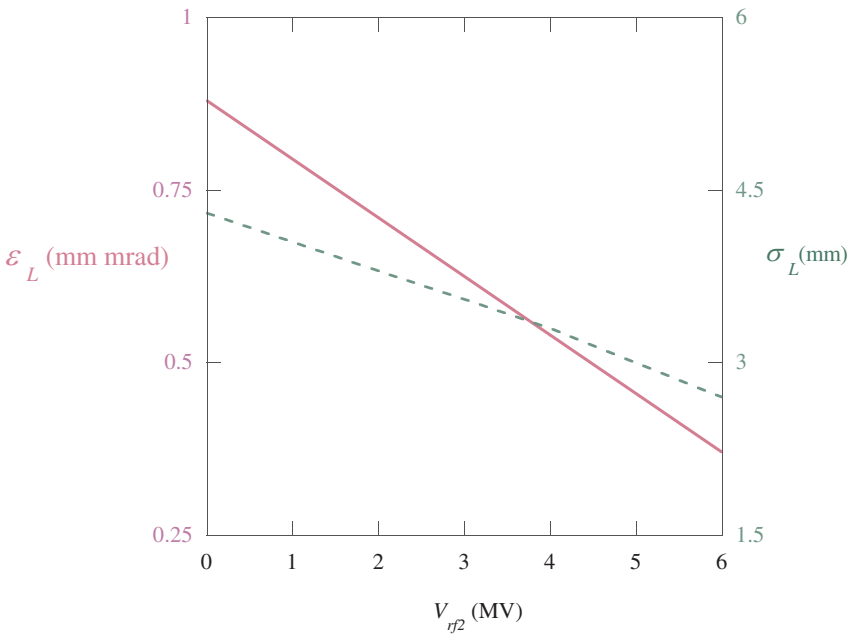

FIG. 15. (Color) Emittance (full pink line) and energy spread (green dashed line) as a function of the second rf cavity voltage for $V_{\mathrm{rf} 1}=4 \mathrm{MV}$. ring in which the drift is monotonic and with constant derivative, but in a real ring the physical length of the section where the bunch is longer can be a large fraction of the total circumference.

The case with nonmonotonic $R_{1}(s)$ has even more free parameters, and a wide range of regimes can be obtained in the same ring by tailoring the behavior of the functions $R_{1,2}(s)$.

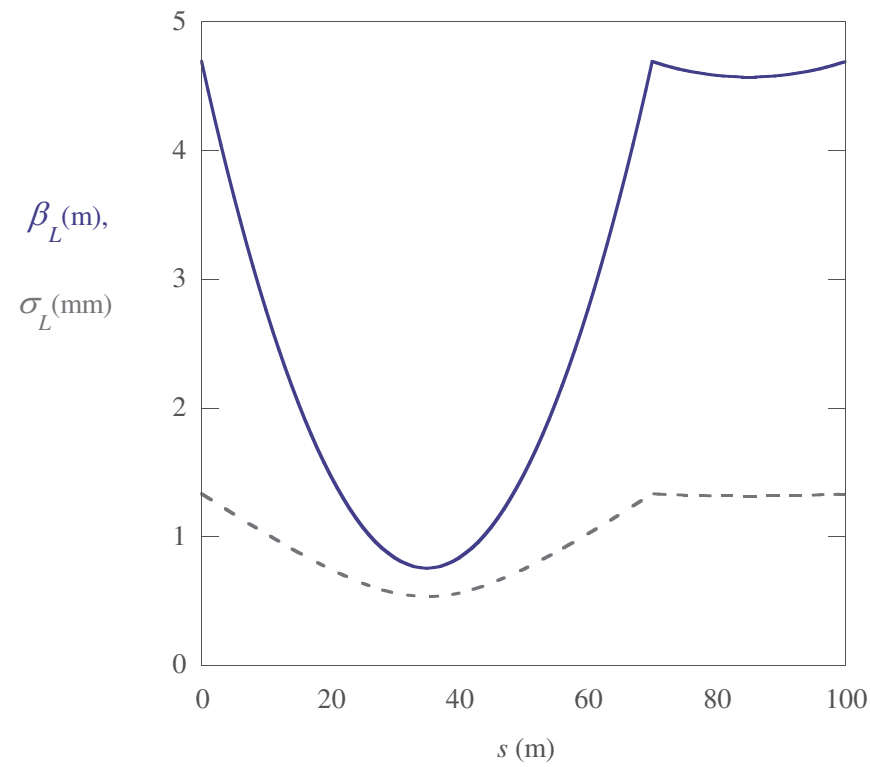

FIG. 16. (Color) Beta function (full blue line) and bunch length (dashed gray line) along the ring for monotonic $R_{1}(s)$ with two equal cavities $\left(U_{1}=U_{2}\right)$ at a drift distance $R_{3}=a_{c} L / 3$. 
All the considerations can be generalized to a ring with several rf systems.

\section{CONCLUSIONS}

Bunch length in storage rings is usually constant along the circumference. High synchrotron tunes regimes can introduce a modulation in $\sigma_{L}$, whose principle was initially sketched by Piwinski in late 1960s [1], but has never been implemented up to now in any real machine. In the framework of superfactory studies the strong rf focusing principle has been recently proposed [3] to obtain very short bunches at the interaction point, while keeping the average bunch length above the microwave instability threshold, by using high momentum compaction lattices and high $\mathrm{rf}$ voltage derivative.

This paper shows the possibility of obtaining bunch length modulation regimes also with low synchrotron tune, and compares this novel regime with the strong rf focusing one. A lattice in which the absolute value of the dispersion function is high in the dipoles, but with different sign in different zones of the ring, plus a high rf voltage derivative, will give rise to drifting of the longitudinal phase space, and therefore bunch length modulation, while keeping a small momentum compaction and low synchrotron tune. The modulation factor in this case can be strongly increased by approaching the isochronicity condition, thus relaxing the condition on the necessary rf voltage derivative.

A difference between the high and the low synchrotron tune cases is the position along the ring of the minimum bunch length, which in the first case is far from the cavity, while in the second case is near the rf cavity, or even at the cavity position for the isochronous limit. In all cases an enhancement of the longitudinal emittance and of the natural equilibrium energy spread appears as the modulation regime is approached.

The use of more than one rf system can change the bunch length behavior along the ring with a wide range of possibilities.

Such regimes could be used in colliders as mentioned before, or in coherent synchrotron radiation (CSR) sources, in order to produce the stable CSR in limited zones of the ring.

Only linear single particle dynamics is here discussed. The feasibility of a regime with bunch length modulation requires a coordinate choice of transverse and longitudinal working points, since the synchrobetatron resonances are enhanced by this regime [4] and of course a detailed analysis of how collective effects interfere with the natural longitudinal distribution here described.

\section{ACKNOWLEDGMENTS}

I thank my colleagues and friends Alessandro Gallo, Andrea Ghigo, Mario Serio, and Mikhail Zobov for the enlightening discussions on the physics and the mathematics of the subject.

[1] A. Piwinski, Nucl. Instrum. Methods 72, 79 (1969).

[2] A. Hofmann (private communication).

[3] A. Gallo, P. Raimondi, and M. Zobov, physics/0404020.

[4] C. Biscari, D. Alesini, G. Benedetti, M.E. Biagini, R. Boni, M. Boscolo, A. Clozza, G. Delle Monache, G. Di Pirro, A. Drago, A. Gallo, A. Ghigo, S. Guiducci, M. Incurvati, E. Levichev, C. Ligi, F. Marcellini, G. Mazzitelli, C. Milardi, L. Pellegrino, P. Piminov, M. A. Preger, P. Raimondi, R. Ricci, C. Sanelli, M. Serio, F. Sgamma, A. Stecchi, A. Stella, C. Vaccarezza, M. Vescovi, and M. Zobov, in Proceedings of EPAC04, Lucerne, 2004 (EPS-AG, Lucerne, 2004).

[5] H. Wiedemann, Handbook of Accelerator Physics and Engineering (World Scientific, Singapore, 2000).

[6] A. Gallo, D. Alesini, G. Benedetti, M.E. Biagini, C. Biscari, R. Boni, M. Boscolo, A. Clozza, G. Delle Monache, G. Di Pirro, A. Drago, A. Ghigo, S. Guiducci, M. Incurvati, E. Levichev, C. Ligi, F. Marcellini, G. Mazzitelli, C. Milardi, C. Pagani, L. Pellegrino, P. Piminov, M. A. Preger, P. Raimondi, R. Ricci, U. Rotundo, C. Sanelli, M. Serio, F. Sgamma, B. Spataro, A. Stecchi, A. Stella, F. Tazzioli, C. Vaccarezza, M. Vescovi, and M. Zobov, in Proceedings of EPAC04, Lucerne, 2004 (Ref. [4]).

[7] A. W. Chao, J. Appl. Phys. 50, 595 (1979). 